\title{
Stereotactic radiotherapy in the treatment of local recurrences of esophageal cancer
}

\author{
MARCEL MATZENAUER ${ }^{1}$, DAVID VRÁNA ${ }^{1-3}$, ZUZANA VLACHOVÁ $^{1}$, RENE AUJESKY $^{4}$, \\ RADEK VRBA $^{4}$, CESTMIR NEORAL ${ }^{4}$ and BOHUSLAV MELICHAR ${ }^{1,2}$ \\ ${ }^{1}$ Department of Oncology, Faculty of Medicine and Dentistry, Palacky University; \\ ${ }^{2}$ Institute of Molecular and Translational Medicine, Faculty of Medicine and Dentistry, Palacky University, \\ University Hospital Olomouc, 77900 Olomouc; ${ }^{3}$ Toxicogenomics Unit, National Institute of Public Health, 10042 Prague; \\ ${ }^{4}$ Department of Surgery, Faculty of Medicine and Dentistry, Palacky University, 77900 Olomouc, Czech Republic
}

Received May 4, 2016; Accepted November 10, 2016

DOI: $10.3892 / \mathrm{ol} .2017 .5605$

\begin{abstract}
Esophageal cancer (EC) consists of tumors with a generally poor prognosis, and treatment options for patients with disease recurrence are extremely limited. Due to this poor patient prognosis, the possible treatment toxicity should be carefully balanced against its potential benefit and patient quality of life. Stereotactic body radiotherapy (SBRT) is a rapidly expanding novel technique combining a short treatment time together with high local efficacy and an acceptable toxicity profile. There are no publications thus far presenting data regarding the usage of SBRT utilizing a conventional linear accelerator in locally recurrent EC patients. In the present study, 2 patients with recurrent EC in the neck lymph nodes were treated by SBRT in the Department of Oncology, University Hospital Olomouc, Czech Republic. The treatment dose was 30 and 40 Gy in 5 daily fractions, with a prescribed dose to 65 and $81 \%$ isodose, for each patient respectively, utilizing a volumetric arc therapy technique, a 6-MV photon beam and an Elekta Synergy linear accelerator. The treatment was delivered without any unintentional treatment interruptions and without any treatment-related acute toxicity. The maximum dose in the patients was 45.9 and $49.2 \mathrm{~Gy}$, respectively. The maximum doses for the surrounding major blood vessels were 35.4 and $45.7 \mathrm{~Gy}$, respectively. Maximum doses to the trachea and the esophagus in the first patient were 32.6 and $27.0 \mathrm{~Gy}$. In the second patient, these doses were not clinically significant. SBRT utilizing linear accelerators should be considered in patients with localized recurrent EC, offering the patients the chance for local control with minimal treatment toxicity.
\end{abstract}

Correspondence to: Dr David Vrána, Department of Oncology, Faculty of Medicine and Dentistry, Palacky University, I. P. Pavlova 6, 77900 Olomouc, Czech Republic

E-mail: davvrana@gmail.com

Key words: esophageal cancer, stereotactic radiotherapy, recurrence, chemotherapy

\section{Introduction}

Patients with esophageal cancer (EC), regardless of histology (squamous or adenocarcinoma), have a poor prognosis, with a reported 3-year overall survival rate of between 40 and $50 \%$ (1). Despite recent advances in radiation treatments and the field of medical oncology during recent decades, the treatment of EC has not changed significantly, and the results remain disappointing. The RTOG 85-01 trial demonstrated that patients treated with chemoradiation have a significantly better outcome compared with patients treated with radiotherapy alone (2). At the same time, the Intergroup 0123 (RTOG 9405) trial provided evidence that dose escalation (increasing the dose from 50.4 to $64.8 \mathrm{~Gy}$ ) has no benefit in EC patients (3). The reasons for these negative findings remain unclear, however, the treatment-related toxicity (specifically lung toxicity) of the higher dose may be the cause of the inferior prognosis of patients randomized in the arm with a higher radiation dose. Moreover, the CROSS trial showed the benefit of even lower doses of radiation using 41.4 Gy in 23 fractions, administered with weekly carboplatin and paclitaxel chemotherapy (4). As a complete response is obtained in only $\sim 30 \%$ of patients treated with concomitant chemoradiation, subsequent esophagectomy appears to be necessary (5). However, surgery alone has conferred a worse outcome compared with tri-modality treatment (6). Patient selection for this treatment should be carefully performed, particularly with regard to patient nutrition and performance status. Tri-modality treatment, i.e., neoadjuvant chemoradiation followed by surgery, represents the current standard of care for patients with localized EC. However, this treatment is associated with significant morbidity (7).

There are no clear recommendations regarding the frequency and imaging techniques to be used during the follow-up of EC patients following radical therapy. The monitoring strategies range from tri-monthly upper gastrointestinal endoscopy together with computed tomography (CT), to total omission of the follow-up, referring the patients to a general practitioner and waiting for clinically manifested disease relapse. There is no evidence of any benefit of using imaging modalities, endoscopy or circulating tumor markers for the follow-up of patients and a number of medical or radiation 
oncologists have argued against the use of any follow-up due to the extra cost, without any advantage of an early diagnosis of EC recurrence. In fact, there is only limited evidence that salvage treatments prolong patient survival (8).

The majority of EC patients following radical therapy will eventually experience disease relapse. Several treatment options are available for these patients. In the case of local relapse in the esophagus, surgery represents a potentially curative approach. Intraluminal brachytherapy and chemotherapy are only palliative treatments (9). Stenting is another effective method of palliation (10). If the tumor relapse occurs outside the esophagus in the form of only limited oligometastatic disease, such as occurs in the lymph nodes, then surgery or palliative chemotherapy could again be considered. Radiotherapy using conventional fractionation is frequently not feasible due to the dose constraints after the primary neoadjuvant chemoradiation (11). Subsequent to further progression or in the case of metastatic disease when local therapy is not possible, palliative chemotherapy based on cisplatin, carboplatin, 5-fluorouracil or paclitaxel is another option (12).

The use of stereotactic body radiotherapy (SBRT) is rapidly expanding in the treatment of almost all tumor types and anatomical regions. However, data for the use of SBRT in local EC recurrence is currently missing $(13,14)$. SBRT utilizes a high-dose gradient drop off, a limited number of fractions and a high dose per fraction, with a biological equivalent dose usually exceeding 100 Gy. SBRT has the advantage of a high probability of local tumor control and, at the same time, a short treatment duration and limited toxicity, leaving the palliative chemotherapy as an option for a subsequent line of treatment following disease progression. Quality of life in EC patients is extremely important and platinum-based chemotherapy (cisplatin or carboplatin) poses a significant risk of toxicity. The present study concerns the usage of SBRT in patients with the recurrence of esophageal cancer and aims to demonstrate the favorable safety and efficacy of the technique.

\section{Patients and methods}

Tumor volumes. For the purposes of conturing and treatment planning, CTs with a 3-mm slide thickness were obtained for the two patients. Involved lymph nodes were contoured as the gross tumor volume (GTV). The clinical target volume (CTV) was identical to the GTV, assuming no extranodal extension of the disease. The planning target volume (PTV) was created by adding a $3-\mathrm{mm}$ margin to the CTV for possible intrafractional movements. The PTV margin was based on institutional SBRT standards. A 3-point thermoplastic mask was utilized for patient immobilization. Organs at risk (OAR; trachea, major vessels and spinal cord) in the vicinity of the PTV were contoured at least $1 \mathrm{~cm}$ above and below the PTV. The treatment plan was prepared utilizing the Monaco ${ }^{\circledR}$ planning system (Elekta Instrument AB, Stockholm, Sweden) using the Monte Carlo calculation algorithm.

Plan evaluation. In the 2 patients, the local recurrence was outside the high-dose region of previous radiotherapy (refining the high dose as a region with a dose $>30 \mathrm{~Gy}$ ). For the treatment, Elekta Synergy linear accelerators (Elekta Instrument AB) were employed (6-MV photon beam, volumetric arc therapy technique). Doses of $30 \mathrm{~Gy}$ in the first case and $40 \mathrm{~Gy}$ in the second case were prescribed in 5 daily fractions. Since no recommendations or any publications exist regarding the dose for use in the lymph node recurrence of EC, a dose was selected with respect to the dose constraints of surrounding OARs. The dose gradient as a ratio of the volume of a 100 and 50\% isodose was assessed (Paddick) (15), as well as the conformity index (ICRU 83) (16), defined as a ratio of the volume of the $100 \%$ isodose and the volume of the PTV that received the prescribed dose as recommended in ICRU 83. To confirm appropriate patient immobilization and setup, three cone beam CTs were utilized, two prior to and one subsequent to dose delivery.

\section{Results}

A total of 2 patients with esophageal adenocarcinoma and squamous cell carcinoma, respectively, who were initially treated with concurrent chemoradiation (50 Gy in 25 fractions, along with 3 cycles of cisplatin and 5-fluorouracil chemotherapy), followed by esophagectomy in the first case and observation in the second case, were enrolled in the present study. Surgery was not completed for the second patient due to co-morbidities and a complete response after the neoadjuvant treatment, as confirmed by positron emission tomography combined with CT (PET/CT). Nodal recurrences occurred in the neck region in each case. Disease recurrence in the form of isolated nodal disease with no evidence of other metastases was diagnosed in the 2 patients using PET/CT. As PET/CT is not able to distinguish between the tumor and inflammation, particularly in the case of lymph nodes in the head and neck region where inflammatory lymph nodes are frequent findings, and as the lymph nodes in the patients could not be biopsied by fine-needle aspiration biopsy, a decision was made to perform 3-deoxy-3-[18F]-fluorothymidine PET (FLT-PET). This technique was used to aid the differential diagnosis, as it exhibits a significantly higher positive predictive value for the diagnosis of neoplasia compared with PET/CT. FLT-PET showed uptake in the same lymph nodes, confirming the high suspicion for the presence of metastatic disease. Each patient was discussed during the multidisciplinary team meetings. Due to the significant risk of surgery in each case, it was decided to proceed with SBRT. These conclusions were discussed with the patients and the rationale, practical aspects and potential side effects of radiotherapy were explained to them. Written informed consent for SBRT and publication of the present study was obtained from the two patients. In total, 2 patients, 1 man and 1 woman aged 62 and 57 years, respectively, were treated at the University Hospital Olomouc. Each patient completed the treatment with SBRT at the beginning of 2016. For disease staging purposes, the TNM 7th classification was utilized (17).

The patient characteristics are summarized in Table I. The isodose distribution for the treatment plans are presented in Figs.1 and 2, and show 100 and 50\% isodoses together with contours for the OAR. Due to the location of the recurrences, the doses to the trachea, major vessels and esophagus had to be assessed in the first patient, while only the dose to the major blood vessels had to be evaluated in the second patient. The maximum doses to the trachea, the esophagus and the major vessels in the first patient were 32.6, 27.0 and 35.4 Gy, respectively, and the maximal dose to the major blood vessels was $45.7 \mathrm{~Gy}$ in the 
Table I. Patient characteristics.

\begin{tabular}{lll}
\hline Characteristic & \multicolumn{1}{c}{ Patient 1} & Patient 2 \\
\hline Age at diagnosis, years & 57 & 62 \\
Primary tumor staging & cT3cN0M0 & cTxcN1M0 \\
Histology & Squamous cell carcinoma & Adenocarcinoma \\
Primary treatment & Chemoradiotherapy & Chemoradiotherapy (surgery not indicated \\
& followed by surgery & due to comorbidities) \\
Disease-free interval, months & 18 & 21 \\
Site of recurrence & Lower neck lymph nodes & Neck lymph nodes \\
Number of fractions of SBRT & 5 & 5 \\
Dose prescribed, Gy & 30 & 40 \\
Prescription isodose, $\%$ & 65 & 81 \\
Maximum dose, Gy & 45.9 & 49.2 \\
Dose gradient (Paddick) & 0.17 & 0.12 \\
Conformity index (ICRU 83) & 0.91 & 0.88 \\
Maximum dose to trachea, Gy & 32.6 & Not applicable \\
Maximum dose to major blood vessels, Gy & 35.4 & 45.7 \\
Maximum dose to esophagus, Gy & 27 & Not applicable
\end{tabular}

SBRT, stereotactic body radiotherapy; ICRU, International Commission on Radiation Units.

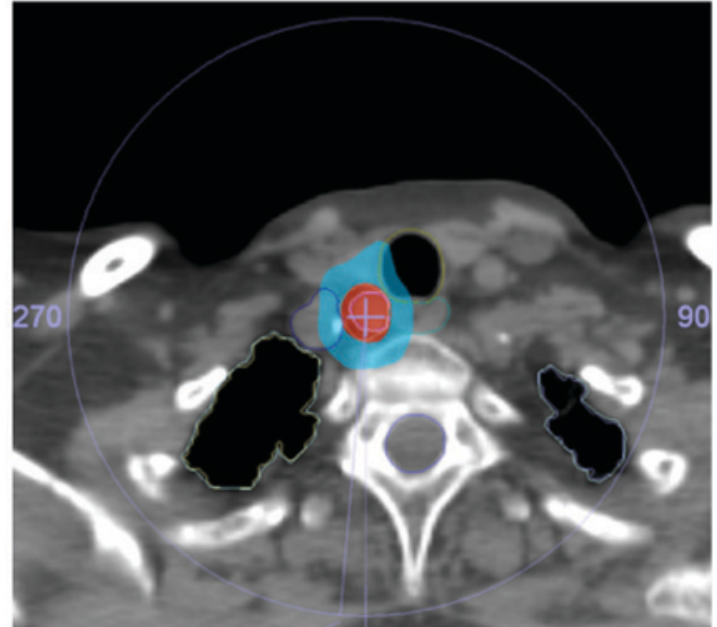

Figure 1. Patient 1 dose distribution. Red, 100\% isodose; blue, 50\% isodose.

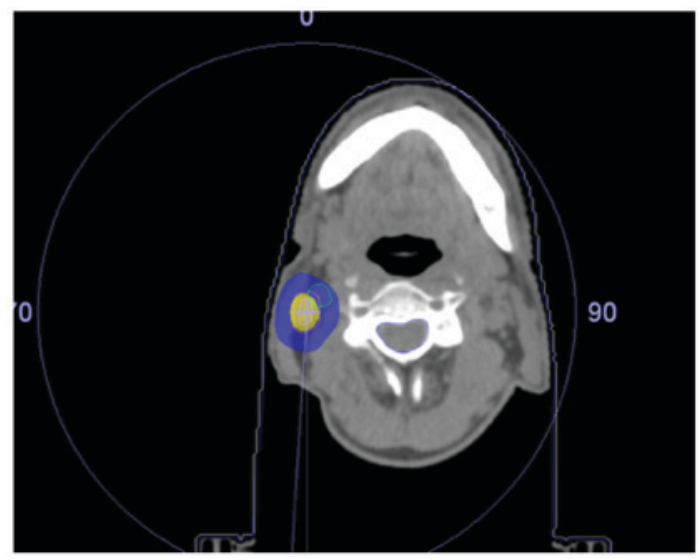

Figure 2. Patient 2 dose distribution. Yellow, 100\% isodose; blue, 50\% isodose. second patient. The doses to the spinal cord, brachial plexus and lungs were not significant for either patient. The dose to the thyroid gland was not specifically evaluated. The maximum dose was $45.9 \mathrm{~Gy}$ in patient 1 and $49.2 \mathrm{~Gy}$ in patient 2. The treatment dose was delivered without any unintentional treatment interruptions and the intrafractional movements during all fractions of radiotherapy were under $3 \mathrm{~mm}$, indicating that a 3 -point thermoplastic mask is appropriate for patient immobilization during SBRT in the neck region. The patients tolerated the treatment well and did not experience any significant treatment-related toxicity during the follow-up.

\section{Discussion}

The treatment options for patients with recurrent EC are limited and the prognosis is poor, with expected survival restricted to months rather than years. Evidence of any convincing activity for a given therapeutic approach to support the selection of an optimal treatment modality is mostly missing. Patient performance status, co-morbidities and the patient's own preference should be considered. In general, there are two treatment options in a case of localized relapse, consisting of either surgical removal of the tumor recurrence, which is often not feasible due to previous radiotherapy and surgery, or systemic palliative chemotherapy. Surgeons are frequently reluctant to attempt a surgical resection in patients with recurrent EC due to the poor prognosis. Chemotherapy based on the combination of cisplatin and 5-fluorouracil has a limited effect on the survival of patients with EC, and the addition of taxanes, such as paclitaxel, has been shown to not significantly affect the outcome (18). The targeted agents ramucirumab and trastuzumab have been introduced into the therapy of patients with metastatic adenocarcinoma of the gastroesophageal junction, but the activity of these drugs is limited $(19,20)$. The data to support the use of immunotherapy 
in patients with primary/recurrent EC is currently limited, although numerous trials are ongoing. In the case of asymptomatic recurrence, there is also an option to observe the patient and intervene at the time of the manifestation of symptoms, taking into account the marginal survival benefit of palliative treatment for recurrent disease. However, this option may not be acceptable for a number of the patients and their attending oncologists. The quality of life of affected patients must always be one of the principal considerations during the decision on the selection of an appropriate treatment strategy.

SBRT utilizing conventional linear accelerators is a rapidly evolving technique that, due to low toxicity and a short overall treatment time, may be considered an ideal therapeutic option for patients with a poor prognosis, such as those with recurrent $\mathrm{EC}$ in whom the balance between quality of life and treatment toxicity should be carefully weighed. The utilization of SBRT has been widely reported in patients with primary lung cancer, primary liver cancer and metastatic disease, and in individuals with recurrences of gynecological tumors and brain metastases, with SBRT quickly becoming a standard institutional treatment worldwide (21-23). However, there are few studies describing the use of SBRT in locally recurrent EC patients without distant metastases, and further studies are required to clarify the optimal treatment approach in this group of patients $(24,25)$.

In conclusion, SBRT is a technically feasible and safe option for patients with locally recurrent EC that provides the possibility of local control and a good quality of life during and after the treatment.

\section{Acknowledgements}

The study was supported by grant IGA_LF_2016_014, provided by Palacky University (Olomouc, Czech Republic).

\section{References}

1. Koshy M, Greenwald BD, Hausner P, Krasna MJ, Horiba N, Battafarano RJ, Burrows W and Suntharalingam M: Outcomes after trimodality therapy for esophageal cancer: The impact of histology on failure patterns. Am J Clin Oncol 34: 259-264, 2011.

2. Cooper JS, Guo MD, Herskovic A, Macdonald JS, Martenson JA Jr, Al-Sarraf M, Byhardt R, Russell AH, Beitler JJ, Spencer S, et al: Chemoradiotherapy of locally advanced esophageal cancer: Long-term follow-up of a prospective randomized trial (RTOG 85-01). Radiation Therapy Oncology Group. JAMA 281: 1623-1627, 1999.

3. Minsky BD, Pajak TF, Ginsberg RJ, Pisansky TM, Martenson J, Komaki R, Okawara G, Rosenthal SA and Kelsen DP: INT 0123 (Radiation Therapy Oncology Group 94-05) phase III trial of combined-modality therapy for esophageal cancer: High-dose versus standard-dose radiation therapy. J Clin Oncol 20 1167-1174, 2002.

4. Shapiro J, van Lanschot JJ, Hulshof MC, van Hagen P, van Berge Henegouwen MI, Wijnhoven BP, van Laarhoven HW, Nieuwenhuijzen GA, Hospers GA, Bonenkamp JJ, et al: Neoadjuvant chemoradiotherapy plus surgery versus surgery alone for oesophageal or junctional cancer (CROSS): Long-term results of a randomised controlled trial. Lancet Oncol 16: 1090-1098, 2015.

5. Van Hagen P, Hulshof MC, van Lanschot JJ, Steyerberg EW, van Berge Henegouwen MI, Wijnhoven BP, Richel DJ, Nieuwenhuijzen GA, Hospers GA, Bonenkamp JJ, et al: Preoperative chemoradiotherapy for esophageal or junctional cancer. N Engl J Med 366: 2074-2084, 2012

6. Tepper J, Krasna MJ, Niedzwiecki D, Hollis D, Reed CE, Goldberg R, Kiel K, Willett C, Sugarbaker D and Mayer R: Phase III trial of trimodality therapy with cisplatin, fluorouracil, radiotherapy, and surgery compared with surgery alone for esophageal cancer: CALGB 9781. J Clin Oncol 26: 1086-1092, 2008.
7. Hoeppner J, Zirlik K, Brunner T, Bronsert P, Kulemann B, Sick O, Marjanovic G, Hopt UT and Makowiec F: Multimodal treatment of locally advanced esophageal adenocarcinoma: Which regimen should we choose? Outcome analysis of perioperative chemotherapy versus neoadjuvant chemoradiation in 105 patients. J Surg Oncol 109: 287-293, 2014.

8. Ford HE, Marshall A, Bridgewater JA, Janowitz T, Coxon FY, Wadsley J, Mansoor W, Fyfe D, Madhusudan S, Middleton GW, et al: Docetaxel versus active symptom control for refractory oesophagogastric adenocarcinoma (COUGAR-02): An open-label, phase 3 randomised controlled trial. Lancet Oncol 15: 78-86, 2014.

9. Laskar SG, Lewis S, Agarwal JP, Mishra S, Mehta S and Patil P: Combined brachytherapy and external beam radiation: An effective approach for palliation in esophageal cancer. J Contemp Brachytherapy 7: 453-461, 2015.

10. Rabenstein T: Palliative endoscopic therapy of esophageal cancer. Viszeralmedizin 31: 354-359, 2015.

11. Kim YS, Lee CG, Kim KH, Kim T, Lee J, Cho Y and Koom WS: Re-irradiation of recurrent esophageal cancer after primary definitive radiotherapy. Radiat Oncol J 30: 182-188, 2012.

12. Ilson DH: Esophageal cancer chemotherapy: Recent advances. Gastrointest Cancer Res 2: 85-92, 2008.

13. Crane $\mathrm{CH}$ : Hypofractionated ablative radiotherapy for locally advanced pancreatic cancer. J Radiat Res 57 (Suppl 1): i53-i57, 2016.

14. Pontoriero A, Iatì G, Aiello D and Pergolizzi S: Stereotactic radiotherapy in the retreatment of recurrent cervical cancers, assessment of toxicity, and treatment response: Initial results and literature review. Technol Cancer Res Treat 15: 759-765, 2016.

15. Paddick I and Lippitz B: A simple dose gradient measurement tool to complement the conformity index. J Neurosurg 105 (Suppl): S194-S201, 2006.

16. International Commission on Radiation Units and Measurements: Prescribing, Recording and Reporting Intensity-Modulated Photon-Beam Therapy (IMRT) (ICRU Report 83). J ICRU 10: 1-10618, 2010.

17. Brierley JD, Gospodarowicz MK and Wittekind C (eds): TNM Classification Of Malignant Tumours, 7th edition. Wiley-Blackwell, pp62-66, 2009.

18. Mizota A, Shitara K, Kondo C, Nomura M, Yokota T, Takahari D, Ura T and Muro K: A retrospective comparison of docetaxel and paclitaxel for patients with advanced or recurrent esophageal cancer who previously received platinum-based chemotherapy. Oncology 81: 237-242, 2011.

19. Wilke H, Muro K, Van Cutsem E, Oh SC, Bodoky G, Shimada Y, Hironaka S, Sugimoto N, Lipatov O, Kim TY, et al: Ramucirumab plus paclitaxel versus placebo plus paclitaxel in patients with previously treated advanced gastric or gastro-oesophageal junction adenocarcinoma (RAINBOW): A double-blind, randomised phase 3 trial. Lancet Oncol 15: 1224-1235, 2014.

20. Bang YJ, Van Cutsem E, Feyereislova A, Chung HC, Shen L, Sawaki A, Lordick F, Ohtsu A, Omuro Y, Satoh T, et al: Trastuzumab in combination with chemotherapy versus chemotherapy alone for treatment of HER2-positive advanced gastric or gastro-oesophageal junction cancer (ToGA): A phase 3 , open-label, randomised controlled trial. Lancet 376: 687-697, 2010.

21. Kirichenko A, Gayou O, Parda D, Kudithipudi V, Tom K, Khan A, Abrams P, Szramowski M, Oliva J, Monga D, et al: Stereotactic body radiotherapy (SBRT) with or without surgery for primary and metastatic liver tumors. HPB (Oxford) 18: 88-97, 2016.

22. Matzenauer M, Vrana D, Vlachova Z, Cwiertka K, Kalita O and Melichar B: Radiotherapy management of brain metastases using conventional linear accelerator. Biomed Pap Med Fac Univ Palacky Olomouc Czech Repub 160: 412-416, 2016.

23. Vrána D, Študentová H, Matzenauer M, Vlachová Z, Cwierka K, Gremlica D and Kalita O: Treatment of brain metastases of renal cell cancer with combined hypof ractionated stereotactic radiotherapy and whole brain radiotherapy with hippocampal sparing. Oncology Lett 11: 3777-3781, 2016.

24. Santeufemia DA, Tumolo S, De Paoli A, Lo Re G, Boz G, Miolo GM, Baresic T, Basso SM and Innocente R: Chemo/tomotherapy stereotactic body radiation therapy (chemo/SBRT) for the salvage treatment of esophageal carcinoma following trimodality therapy: A case report. Tumori 98: 143e-145e, 2012.

25. Russo JK and Rosen L: TomoTherapy stereotactic body radiation therapy (SBRT) for the salvage treatment of locally recurrent esophageal adenocarcinoma following trimodality therapy: A case report. Tumori 97: 406-410, 2011. 